\title{
Subcellular concentrations of sugar alcohols and sugars in relation to phloem translocation in Plantago major, Plantago maritima, Prunus persica, and Apium graveolens
}

\author{
Jan Nadwodnik · Gertrud Lohaus
}

Received: 4 August 2007 / Accepted: 7 December 2007 / Published online: 9 January 2008

(C) The Author(s) 2007

\begin{abstract}
Sugar and sugar alcohol concentrations were analyzed in subcellular compartments of mesophyll cells, in the apoplast, and in the phloem sap of leaves of Plantago major (common plantain), Plantago maritima (sea plantain), Prunus persica (peach) and Apium graveolens (celery). In addition to sucrose, common plantain, sea plantain, and peach also translocated substantial amounts of sorbitol, whereas celery translocated mannitol as well. Sucrose was always present in vacuole and cytosol of mesophyll cells, whereas sorbitol and mannitol were found in vacuole, stroma, and cytosol in all cases except for sea plantain. The concentration of sorbitol, mannitol and sucrose in phloem sap was 2- to 40-fold higher than that in the cytosol of mesophyll cells. Apoplastic carbohydrate concentrations in all species tested were in the low millimolar range versus high millimolar concentrations in symplastic compartments. Therefore, the concentration ratios between the apoplast and the phloem were very strong, ranging between 20- to 100-fold for sorbitol and mannitol, and between 200and 2000-fold for sucrose. The woody species, peach, showed the smallest concentration ratios between the cytosol of mesophyll cells and the phloem as well as between the apoplast and the phloem, suggesting a mixture of apoplastic and symplastic phloem loading, in contrast to the herbal plant species (common plantain, sea plantain, celery) which likely exhibit an active loading mode for sorbitol and mannitol as well as sucrose from the apoplast into the phloem.
\end{abstract}

J. Nadwodnik · G. Lohaus ( $\square)$

Albrecht-von-Haller-Institut für Pflanzenwissenschaften,

Biochemie der Pflanze, Universität Göttingen,

Untere Karspüle 2, 37073 Gottingen, Germany

e-mail: glohaus@gwdg.de
Keywords Apium $\cdot$ Mannitol $\cdot$ Phloem $\cdot$ Plantago . Prunus · Sorbitol

Abbreviations
FW $\quad$ Fresh weight
HPLC

\section{Introduction}

In order to understand carbon partitioning, the regulation of photosynthesis, phloem loading, or sink-source interactions, it is important to know how photoassimilates are distributed between the subcellular compartments of mesophyll cells of source leaves. Much of the information concerning subcellular metabolite concentrations is derived from studies of species such as spinach, barley, tobacco, and potato. In such plant species carbohydrates assimilated from $\mathrm{CO}_{2}$ are exported into the phloem exclusively in the form of sucrose (Riens et al. 1991; Winter et al. 1992; Lohaus et al. 1998). However, it has been shown that in several plant species, sugar alcohols like mannitol, sorbitol or dulcitol are also translocated in addition to sucrose (Zimmermann and Ziegler 1975). The advantage of transporting sucrose and sugar alcohols is that these compounds are highly soluble and chemically inert. In addition to functioning as a transport form for reduced carbon, sugar alcohols have been also shown to serve as storage for reduced carbon, as compatible solute synthesized in response to abiotic or biotic stress, or as osmoprotectants. It has been estimated that up to $30 \%$ of the carbon fixed by plants on earth may be present in the form of polyols (Bieleski 1982).

Mannitol is the most-widely distributed sugar alcohol and has been reported in more than 100 species of vascular plants, including most species of the Apiaceae (celery, 
parsley, and carrot), Oleaceae (olive, privet), Rubiaceae (coffee), and Scrophulariaceae (snapdragon) (Zimmermann and Ziegler 1975; Bieleski 1982). In higher plants, mannitol is synthesized by the action of an NADPH-dependent mannose-6-phosphate reductase that catalyzes the conversion of mannose-6-phosphate to mannitol-1-phosphate, followed by dephosphorylation by a phosphatase (Rumpho et al. 1983). The activity of mannose-6-phosphate reductase was only observed in mature leaves. In different plant species, mannitol synthesis occurs simultaneously with either sucrose synthesis, as in celery (Rumpho et al. 1983), or with raffinose oligosaccharide synthesis, as in Oleaceae (Zimmermann and Ziegler 1975). To date, there are only few reports on the intracellular distribution of mannitol (Keller and Matile 1989; Moore et al. 1997) and these studies do not include measurements of phloem concentrations of mannitol.

Another sugar alcohol present in higher plants is sorbitol. Sorbitol is a major photoassimilate translocated in addition to sucrose in the phloem of woody Rosaceae, including all members of the economically important genera Malus (apple), Pyrus (pear) and Prunus (stone fruits such as peach, cherry, plum and apricot; Zimmermann and Ziegler 1975; Bieleski 1982; Moing et al. 1997). Sorbitol is synthesized in source organs from glucose-6-phosphate by the activity of aldose-6-P-reductase (Negm and Loescher 1981) converting glucose-6-phosphate to sorbitol-6-phosphate. Sorbitol-6-phosphate is further converted to sorbitol by a specific phosphatase. Sinks have little or no capacity to synthesize sugar alcohols like mannitol or sorbitol (Loescher and Everard 1996). To our knowledge, there is only one report on the intracellular distribution of sorbitol in leaf cells (Moore et al. 1997) and with the exception of peach (Moing et al. 1997) there are no data on polyol concentrations in the phloem sap of sugar-alcohol-translocating species.

In addition to mannitol and sorbitol, most plant species also contain the cyclitol myo-inositol (Zimmermann and Ziegler 1975) which is also produced from glucose-6phosphate (Loewus and Loewus 1983). Myo-inositol and its derivatives are involved in membrane formation, cell wall synthesis, seed germination, and hormone responses as well as stress responses (Loewus and Loewus 1983). As an integral part of galactinol, myo-inositol also acts as a carrier of activated galactose that is transferred to sucrose, yielding raffinose and its polymeric derivates of higher order.

Knowledge of concentrations of sugar alcohols and sugars in subcellular compartments of leaf cells and in the phloem is important to understand possible modes of phloem loading. Therefore, we examined the subcellular concentrations of sorbitol, mannitol, myo-inositol, hexoses and sucrose in peach ( $P$. persica, Rosaceae), celery
(A. graveolens, Apiaceae), common plantain (P. major, Plantaginaceae), and sea plantain ( $P$. maritima, Plantaginaceae) as well as the corresponding concentrations in the apoplast and in the phloem sap. Celery and common plantain were selected because much of what is known about the transport of mannitol and sorbitol has come from studies of these plant species, and more recently, the relevant transporters for sucrose as well as for sugar alcohols were cloned from these species. In addition, sea plantain and peach were selected because both species also translocate sorbitol but the former belongs to the group of halophytes and the latter to the group of woody plants. In this manner, different groups of sorbitol-translocating plants are included in the comparison. The sugar alcohols and sugars listed above are the main soluble carbohydrates in these plants. Based on the subcellular distribution, we calculated the concentration ratios of soluble carbohydrates between the different subcellular compartments as well as in compartments which are involved in phloem loading. In combination with results from morphological studies and data about sucrose and polyol transporters available in the literature the results are compared to predictions based on models of symplastic and apoplastic phloem loading that are currently discussed in the field.

\section{Material and methods}

\section{Materials}

Plants of A. graveolens var dulce cv Vert d'Elne (celery; supplied by R. Lemoine, University of Poitiers cedex, France), $P$. major (common plantain; supplied by N. Sauer, University of Erlangen, Germany) and P. maritima (sea plantain; supplied by N. Sauer, University of Erlangen, Germany) were grown in 2-1 pots in compost soil in a green-house with supplemental illumination at a $15 \mathrm{~h}$ photoperiod and a $23 / 18^{\circ} \mathrm{C}$ day-night thermoperiod. Two-month-old plants were taken for the experiments. P. persica (peach), cv. Red Haven (Jenssen, Göttingen, Germany) was grown in 5-1 pots in compost soil in a green house. Three-year-old plants were used for the experiments. Leaf samples were harvested in May and June.

Non-aqueous fractionation of leaf tissue

Leaves were cut from the plants after $9 \mathrm{~h}$ of the light period. The middle rib and larger veins were removed, and the samples were ground to a fine powder in liquid nitrogen in a precooled mortar. The leaf tissue was lyophilized at $-25^{\circ} \mathrm{C}$. The dry leaf powder was suspended in $20 \mathrm{ml}$ of heptane:tetrachloroethylene mixture (density $1.27 \mathrm{~g} \mathrm{ml}^{-1}$, 
the solvents were dried and stored over molecule sieve beads from Merck, Darmstadt, Germany) and ultrasonicated with 5-s pulses and 5-s breaks for a total sonication period of $95 \mathrm{~s}$ (Cell Disrupter B15, Bronson Sonifer). The suspension was then poured through a polyester monolen sieve with a pore size $<40 \mu \mathrm{m}$, diluted 3 -fold with heptane, and centrifuged for $10 \mathrm{~min}$ at 2,200 $\mathrm{g}$ (Centrifuge 5810R, Eppendorf). The clear supernatant was discarded and the sediment resuspended in $3 \mathrm{ml}$ of a heptane:tetrachloroethylene mixture (density $1.27 \mathrm{~g} \mathrm{ml}^{-1}$ ). Two $200 \mu \mathrm{l}$ aliquots were withdrawn for the determination of enzyme activity and carbohydrates in the unfractionated material. Two milliliter of the remaining material were added to an exponential heptane-tetrachlorethylene gradient with a density difference between 1.27 and $1.48 \mathrm{~g} \mathrm{ml}^{-1}$ for common plantain and sea plantain, and between 1.27 and $1.45 \mathrm{~g} \mathrm{ml}^{-1}$ for peach and celery. The bottom of the centrifugation tube contained a $2 \mathrm{ml}$ cushion of a heptane:tetrachlrethylene mixture with the highest density $\left(1.58 \mathrm{~g} \mathrm{ml}^{-1}\right)$. After centrifugation $(25,000 \mathrm{~g}, 2.5 \mathrm{~h})$ six or seven fractions were collected from the centrifuge tube content. Each of these fractions was divided into two portions, one for assay of marker enzymes and the other for assay of carbohydrates. NADP-glycerine aldehyde phosphatase, PEP-carboxylase and $\alpha$-mannosidase were taken as marker enzymes for stroma, cytosol and vacuole. Chloroplastic material (including stroma) appeared in the lower region of the gradient, the cytosolic compartment was found to be enriched in the middle region of the gradient, whereas vacuolar material was mainly found in the fraction of highest density. Chloroform methanol extracts were prepared from the aliquots for the determination of the carbohydrate concentrations (see next section: "Extraction of sugars and sugar alcohols from non-aqueous fractions").

For the evaluation of the subcellular distribution of sugars and sugar alcohols between the stromal, cytosolic, and vacuolar compartment, a calculation procedure according to Riens et al. (1991) was used. This calculation method is based on the assumption that the metabolites are confined to the three compartments as indicated by the corresponding marker enzymes. The evaluation is done by a computer program testing all possible cases for the distribution of a certain metabolite between the three compartments, using increments of $1 \%$; for example: (1) vacuole $100 \%$, stroma $0 \%$, and cytosol $0 \%$; (2) vacuole $99 \%$, stroma $1 \%$, and cytosol $0 \%$; (3) vacuole 99\%, stroma $0 \%$, and cytosol $1 \%$. There are 5,151 possibilities for the distribution of a metabolite between the three compartments and the program uses possibility yields to calculate the best fit (agreement) with the experimental results. To avoid the results being falsified by analytical errors, the calculations are based on mean values obtained from measurements of at least five independent density gradient fractionations.
Extraction of sugars and sugar alcohols from non-aqueous fractions

Chloroform:methanol extracts were prepared to extract sugars and sugar alcohols from samples after non-aqueous fraction. The aliquots (see above) were dried and $5 \mathrm{ml}$ chloroform:methanol $(3: 7, \mathrm{v} / \mathrm{v})$ was added to the powder. The samples were homogenized until completely thawed and kept on ice for $30 \mathrm{~min}$. The homogenate was then extracted twice with $3 \mathrm{ml}$ water. The aqueous phases were combined and evaporated in a rotary evaporator. The dried residue was dissolved in $0.7 \mathrm{ml}$ ultrapure water (Millipore), syringe-filtrated $(0.45 \mu \mathrm{m}$ cellulose-acetate; Schleicher and Schuell, Dassel, Germany) and stored at $-80^{\circ} \mathrm{C}$.

\section{Collection of sieve tube sap}

Sieve tube sap was obtained from severed stylets of the green-peach aphid, Myzus persicae (Sulz.). About 10 aphids were caged for about $5 \mathrm{~h}$ on the leaf. Their stylets were cut by a laser beam (Lohaus et al. 1995). The exuding phloem sap (10-500 nl) was collected in micro-capillaries (total volume $0.5 \mu \mathrm{l}$ ) and the volume of the exudate was determined by measuring the length occupied by the solution. Evaporation of the phloem sap was prevented by bringing the front edge of the capillary in close contact with the leaf surface and surrounding the end with a plastic cap. The humidity around the capillary was about $80 \%$. Under these conditions no evaporation from reference capillaries was detectable. The samples were ejected into $50 \mu \mathrm{l}$ of distilled sterile water and stored at $-80^{\circ} \mathrm{C}$.

Extraction of apoplastic washing fluids from leaves

Leaves were cut $7-8 \mathrm{~h}$ after the onset of illumination and carefully washed with deionized water. Leaves were placed into a syringe filled with $40 \mathrm{ml}$ deionized water and infiltrated by pulling the plunger, producing a reduced pressure of approximately $20 \mathrm{kPa}$. Thereafter, intact leaves were blotted dry, positioned with the xylem wound up in a $10 \mathrm{ml}$ vessel and centrifuged immediately at $200 \mathrm{~g}$, for $4 \mathrm{~min}$ at $4^{\circ} \mathrm{C}$.

Due to the fact that infiltration of the apoplastic air space leads to a dilution of the apoplastic fluid, the solute concentrations in the apoplastic washing fluid were corrected by the ratio volume of the infiltration solution (which corresponds to the volume of the apoplastic gas space $V_{\text {gas }}$ ) to the volume of the apoplastic water space $\left(V_{\text {water }}\right)$. The ion concentration in the apoplast was calculated by multiplying the ion concentration in the apoplastic washing fluid by the dilution factor $\left(=\left(V_{\text {water }}+V_{\text {gas }}\right) / V_{\text {water }}\right)$. The mean dilution factor of several $\mathrm{C} 3$ herbal dicot plant species is about six (Lohaus et al. 2001). Therefore, the concentrations of the apoplastic fluid were diluted about 6-fold by the infiltration 
procedure in common plantain, sea plantain and celery. The dilution factor in peach was only 2.7 because the apoplastic water space was $17 \%$, expressed as percentage of total leaf water content (Moing et al. 1997) which corresponds to about $113 \mu \mathrm{g} \mathrm{g}^{-1}$ leaf water space (Table 3), and the apoplastic gas space was $190 \mu \mathrm{l}(\mathrm{g} \mathrm{FW})^{-1}$ (Table 3).

Cellular contamination of apoplastic washing fluids was quantified by comparing the activity of malate dehydrogenase in the apoplastic wash fluid with that in leaf extracts (Lohaus et al. 2001). According to this test, cellular crosscontamination was always below $0.1 \%$.

\section{Metabolite analysis}

Sugars and sugar alcohols in the samples after non-aqueous fractionation, apoplastic washing fluids, and phloem sap were analyzed by HPLC. An ion exchange column (CarboMA10; Dionex Corp, Sunnyvale, CA, USA) was eluted isocratically with $600 \mathrm{mM} \mathrm{NaOH}\left(0.4 \mathrm{ml} \mathrm{min}^{-1}\right)$ buffer for $60 \mathrm{~min}$. Sugars were detected by a pulse amperometric detector with a gold electrode (ESA, Model 5200, Coulochem II, Bedford, MA, USA). Pulse setting was at 50, 700 and $-800 \mathrm{mV}$ for 500,540 and $540 \mathrm{~ms}$, respectively. Sugar standards were measured daily, and plant samples were diluted sufficiently to provide signals within the linear range of the detector response $(50-500 \mu \mathrm{M})$. The metabolite amount was calculated from the peak area using Peaknet 5.1 software (Dionex, Idstein, Germany).

Electron microscopy and determination of subcellular volumes

Source leaves were cut into pieces of approximately $2 \mathrm{~mm}^{2}$ and prefixed in $5 \%$ glutaraldehyde $(\mathrm{v} / \mathrm{v}), 5 \mathrm{CaCl}_{2}$, and $50 \mathrm{mM}$ sodium-cacodylate buffer, $\mathrm{pH}$ 7.0. After $3 \mathrm{~h}$ at $4^{\circ} \mathrm{C}$, the fixed slices were washed four times for 15 min each in cold $50 \mathrm{mM}$ sodium-cacodylate buffer, $\mathrm{pH} 7.0$, fixed in $2 \%$ $(\mathrm{w} / \mathrm{v})$ osmium tetraoxide in cacodylate buffer and $0.8 \%(\mathrm{w} / \mathrm{v})$ $\mathrm{K}_{4} \mathrm{Fe}(\mathrm{CN})_{6}$ for $3 \mathrm{~h}$ at $4^{\circ} \mathrm{C}$. The samples were washed five times in distilled water and stained with 5\% uranyl acetate. Samples were dehydrated by subsequent incubation in higher concentrations of acetone [30, 50, 70, 90 and $100 \%$ $(\mathrm{w} / \mathrm{v})]$. The samples were then incubated in an acetone/ epoxyresin mix (Spurr 1969) with increasing concentrations of epoxyresin [33, 66 and $100 \%(w / v)]$ for $1 \mathrm{~h}$ per concentration, and overnight and $4 \mathrm{~h}$ at $100 \%(\mathrm{w} / \mathrm{v})$. Sample blocks were than trimmed with a razor and sectioned in an ultra-microtome (Leica Microsystems, Wetzlar, Germany) with a diamond knife. Slices of approximately $60 \mathrm{~nm}$ were placed onto copper grids (Plano, Marburg, Germany), stained with uranylacetate and $0.3 \%$ lead citrate in $\mathrm{NaOH}$ and observed in a transmission electron microscope (EM 400, Philips, Eindhoven, NL).
Electron micrographs of these sections were used for the evaluation of subcellular volumes according the principle of Delesse (1847): "the areal density of profiles on sections is an unbiased estimate of the volume density of structures" (Weibel and Bolender 1973; Winter et al. 1993; Farré et al. 2001). About 50 electron micrographs were taken from 2 independent fixation procedures and from each 6 different leaves. Low magnification and high magnification $(>\mathrm{x} 1,000)$ were used to determine the areas of most compartments. The cross-sectional areas of subcellular compartments were quantified using analysis software $(\mathrm{NIH}$ image, public domain software, developed at US National Institutes of Health, available at http://rsb.info.nih.gov/nihimage/).

The relative volumes (percent of total) were converted to absolute volumes per unit mass by taking into account the water content per mass fresh weight.

\section{Results}

Subcellular distributions of sugars and sugar alcohols in leaf cells

Subcellular distributions of sugar alcohols, mono- and disaccharides were measured by non-aqueous fractionation and HPLC in common plantain, sea plantain, peach and celery. The chosen method is based on the comparison of metabolite and marker enzyme distributions using a threecompartment calculation program (Riens et al. 1991). The method gives highly reproducible results for metabolites which are exclusively located (e.g., hexoses that are predominantly located in the vacuole). A higher variation is found for metabolites located in more than one compartment. The variability is greatest when the proportion found in a particular compartment is low. Farré et al. (2001) estimated that the limit of detection of a compound in a particular compartment is around 5\% of the total amount in the tissue.

HPLC analysis of gradient fractions of common plantain, sea plantain, and peach showed that sucrose, hexoses (glucose and fructose), myo-inositol, and sorbitol were present in leaf cells in these three plants (Fig. 1a-c). Celery contains mannitol instead of sorbitol (Fig. 1d). In all four species the subcellular distribution of sorbitol and mannitol, respectively, was very different from those of hexoses and sucrose (Fig. 1a-d). Sorbitol (in common plantain and peach) and mannitol (in celery) were located in all three compartments, but predominantly in the vacuole (48-73\%), followed by the stroma (19-27\%) and the cytosol (8-25\%). The distribution in sea plantain was different, and sorbitol was found mainly in the vacuole $(88 \%)$ with only small portions in the stroma and in the cytosol. In contrast, in all 

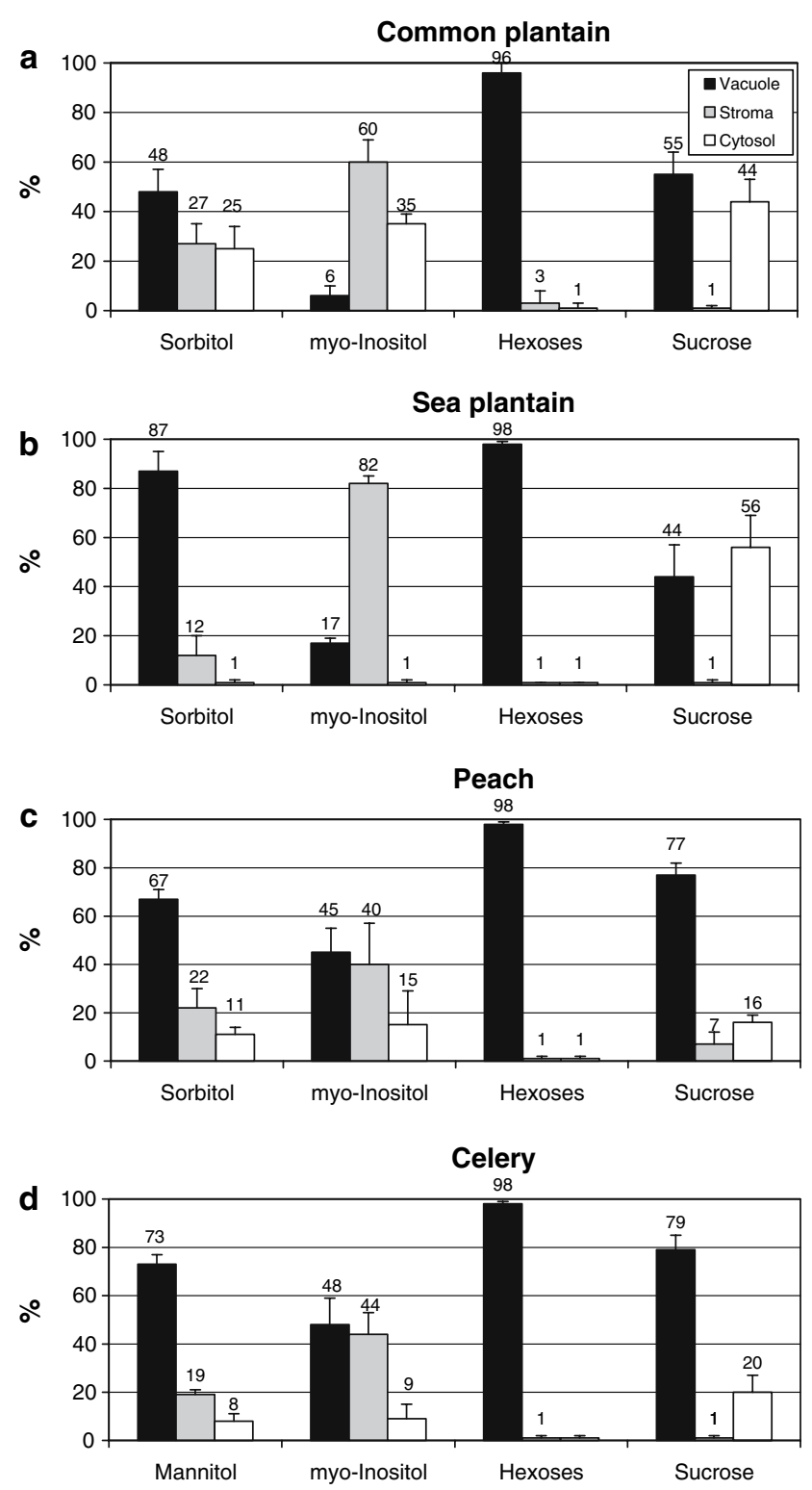

Fig. 1 Percentage distribution of sugars and sugar alcohols among the vacuolar, stromal and cytosolic compartments of leaf cells from a common plantain, b sea plantain, c peach, and d celery. Data represent mean values \pm SD from five to six independent fractionations

four plants glucose and fructose were confined entirely to vacuoles (96-98\%), and in common plantain and sea plantain sucrose was mainly distributed among the vacuolar and the cytosolic compartments (Fig. 1a, b). In peach and celery, a greater proportion of sucrose was found in the vacuolar compartment and a smaller part in the cytosolic compartment (Fig. 1c, d). The cyclitol, myo-inositol, was mostly present in the stroma of common plantain and sea plantain. In peach and celery the proportion was high in the stroma as well as in the vacuole, while the proportion found in the cytosol was always the lowest (Fig. 1c, d).

\section{Subcellular volumes}

The further conversion of the percentage distribution of sugars and sugar alcohols into concentrations requires an estimation of the volumes of the subcellular compartments of mesophyll cells. Leaves contain different tissues but in dicots about $75 \%$ of the aqueous volume of the leaf is occupied by mesophyll cells (Winter et al. 1993, 1994; Leidreiter et al. 1995). Table 1 shows the relative proportions of the vacuolar, chloroplastic and cytoplastic (sum of cytosol, peroxisomes, mitochondria, and nucleus) compartments of TEM micrographs of mesophyll cells. In all plant species analyzed the most voluminous compartment was the vacuole, occupying $68-85 \%$ of the total cell, followed by the chloroplasts $(10-21 \%)$ and the cytoplasm $(5-12 \%)$. The proportion of the vacuole was lowest in the woody plant peach whereas in common plantain and sea plantain mesophyll cells were dominated by the vacuole $(82-85 \%)$. Accordingly, the relative proportions of chloroplasts and cytoplasm were smallest in these plants. The relative proportion of the combined volumes of mitochondria, peroxisomes, and nuclei of the total cytoplasmic volume has been estimated to be about 20\% (Winter et al. 1993, 1994) and the proportion of the stroma volume of the total chloroplast volume about 50\% (Winter et al. 1993, 1994). From the relative proportions mentioned above, the data from Winter et al. $(1993,1994)$ and the average water contents of leaf cells (Table 2) the volumes of the vacuolar, stroma, and cytosolic compartments can be estimated (Table 2).

Subcellular sugar and sugar alcohol concentrations

From the data on leaf carbohydrate contents, the subcelluar volumes, and the distribution of metabolites between the subcellular compartments the corresponding metabolite concentrations in the subcellular compartments were calculated. Sorbitol or mannitol, respectively, made up more than twothird of the total leaf content of soluble carbohydrates in all the plants (Table 3). The concentrations of sorbitol and

Table 1 Relative volumes (\%) of the subcellular compartments within the total volume of mesophyll cells from common plantain, sea plantain, peach and celery

\begin{tabular}{llll}
\hline & Vacuole \% & Chloroplast \% & Cytoplasm \% \\
\hline Common plantain & $82 \pm 5$ & $12 \pm 4$ & $6 \pm 1$ \\
Sea plantain & $85 \pm 5$ & $10 \pm 4$ & $5 \pm 2$ \\
Peach & $68 \pm 7$ & $21 \pm 6$ & $11 \pm 4$ \\
Celery & $72 \pm 5$ & $16 \pm 4$ & $12 \pm 3$ \\
\hline
\end{tabular}

Data were obtained from TEM micrographes $(n=37-42)$. Cytoplasm is defined as cytosol, peroxisomes, nucleus and mitochondria. Mean values $\pm \mathrm{SD}$ 
Table 2 Dry weight $(n=8)$, water and gas space $(n=12)$ of leaves and the volumes of subcellulare compartments of mesophyll cells from common plantain, sea plantain, peach and celery

Mean values \pm SD

Table 3 Whole leaf contents as well as sugar and sugar alcohol concentrations in the vacuolar, stromal and cytosolic compartments of leaf cells from common plantain, sea plantain, peach, and celery

\begin{tabular}{lllll}
\hline & $\begin{array}{l}\text { Common } \\
\text { plantain }\end{array}$ & Sea plantain & Peach & Celery \\
\hline Dry weight $\left(\mathrm{mg} \mathrm{g}^{-1} \mathrm{FW}\right)$ & $147 \pm 9$ & $124 \pm 15$ & $336 \pm 30$ & $140 \pm 8$ \\
Water space $\left(\mu \mathrm{l} \mathrm{g}^{-1} \mathrm{FW}\right)$ & $853 \pm 9$ & $876 \pm 15$ & $664 \pm 30$ & $860 \pm 8$ \\
Gas space $\left(\mu \mathrm{g} \mathrm{g}^{-1} \mathrm{FW}\right)$ & $226 \pm 48$ & $222 \pm 21$ & $190 \pm 44$ & $213 \pm 33$ \\
Volume vacuole $\left(\mu \mathrm{g} \mathrm{g}^{-1} \mathrm{FW}\right)$ & 701 & 743 & 452 & 860 \\
Volume stroma $\left(\mu \mathrm{g} \mathrm{g}^{-1} \mathrm{FW}\right)$ & 50 & 43 & 70 & 68 \\
Volume cytosol $\left(\mu \mathrm{g} \mathrm{g}^{-1} \mathrm{FW}\right)$ & 42 & 38 & 58 & 82 \\
\hline
\end{tabular}

\begin{tabular}{|c|c|c|c|c|}
\hline & \multirow{2}{*}{$\begin{array}{l}\text { Whole leaf } \\
\text { content } \mu \text { mol } \\
(\mathrm{g} \mathrm{FW})^{-1}\end{array}$} & \multicolumn{3}{|c|}{ Concentration $(\mathrm{mM})$} \\
\hline & & Vacuole & Stroma & Cytosol \\
\hline \multicolumn{5}{|l|}{ Common plantain } \\
\hline Sorbitol & $22 \pm 3.1$ & $15 \pm 2.3$ & $118 \pm 34$ & $133 \pm 48$ \\
\hline myo-Inositol & $0.7 \pm 0.2$ & $0.1 \pm 0.1$ & $8.8 \pm 1.3$ & $6.1 \pm 0.7$ \\
\hline Glucose and fructose & $2.4 \pm 1.1$ & $3.2 \pm 0.2$ & $1.4 \pm 2.0$ & $0.6 \pm 0.7$ \\
\hline Sucrose & $1.2 \pm 0.1$ & $0.9 \pm 0.2$ & $0.1 \pm 0.2$ & $12 \pm 2.5$ \\
\hline \multicolumn{5}{|l|}{ Sea plantain } \\
\hline Sorbitol & $73 \pm 9.1$ & $87 \pm 8.0$ & $201 \pm 140$ & $13 \pm 8.4$ \\
\hline myo-Inositol & $0.8 \pm 0.2$ & $0.2 \pm 0.1$ & $15 \pm 0.4$ & $0 \pm 0$ \\
\hline Glucose and fructose & $11 \pm 2.5$ & $15 \pm 0.5$ & $0.2 \pm 0.5$ & $0 \pm 0$ \\
\hline Sucrose & $1.4 \pm 0.2$ & $0.8 \pm 0.3$ & $0.1 \pm 0.1$ & $20 \pm 4.9$ \\
\hline \multicolumn{5}{|l|}{ Peach } \\
\hline Sorbitol & $148 \pm 0.1$ & $220 \pm 15$ & $461 \pm 165$ & $290 \pm 62$ \\
\hline myo-Inositol & $1.5 \pm 0.1$ & $1.5 \pm 0.4$ & $8.5 \pm 3.6$ & $4.1 \pm 3.3$ \\
\hline Glucose and fructose & $30 \pm 6.1$ & $65 \pm 1$ & $5.0 \pm 5.0$ & $5.0 \pm 5.0$ \\
\hline Sucrose & $38 \pm 2.1$ & $64 \pm 4.5$ & $39 \pm 26$ & $106 \pm 15$ \\
\hline \multicolumn{5}{|l|}{ Celery } \\
\hline Mannitol & $108 \pm 121$ & $27 \pm 7.0$ & $294 \pm 30$ & $100 \pm 39$ \\
\hline myo-Inositol & $1.4 \pm 0.1$ & $1.1 \pm 0.3$ & $9.0 \pm 1.9$ & $1.7 \pm 0.9$ \\
\hline Glucose and fructose & $17 \pm 2.0$ & $28 \pm 0.3$ & $2.5 \pm 1.7$ & $2.9 \pm 1.2$ \\
\hline Sucrose & $35 \pm 4.3$ & $45 \pm 3.3$ & $5.2 \pm 3.2$ & $86 \pm 28$ \\
\hline
\end{tabular}

mannitol, respectively, were high in all compartments of peach and celery (Table 3). Most of the sorbitol and mannitol were located in the vacuole (Fig. 1a-d) but due to the smaller volume cytosolic concentrations were calculated to be similar to those in the vacuole, and the highest level was found in the stroma (Table 3). The sorbitol concentration in the cytosol of sea plantain was lower $(13 \mathrm{mM})$ than in the vacuole $(87 \mathrm{mM})$ whereas in common plantain the vacuole showed the lowest sorbitol concentration $(15 \mathrm{mM})$.

In each plant species sucrose was mainly concentrated in the cytosol, followed by the vacuole and was lowest in the stroma, however, concentrations varied widely between the species (cytosol 12-106 mM, vacuole 0.8-64 mM, and stroma 0.1-39 mM; Table 4). These differences between the plant species reflect the differences in the total leaf contents (Table 3). The sucrose content in both plantain species was much lower than in peach and celery.
Of the compartments analyzed the highest concentration of glucose and fructose was consistently found in the vacuole. As the evaluation of the subcellular fraction was performed in increments of $1 \%$, glucose and fructose concentrations found in the cytosol or stroma represent an upper limit of $1 \%$ for these compounds.

The concentration of myo-inositol was highest in the stroma, with intermediate values in the cytosol and lowest in the vacuole (Table 3). Myo-inositol, sorbitol and mannitol, respectively, made up between 90 and $99 \%$ of the total soluble carbohydrates in the stroma of the plant species.

Apoplastic and phloem sap concentrations

Carbohydrates found in the phloem sap of the four plant species investigated were sucrose and mannitol or sorbitol (Table 4). Concentrations were high in all cases, ranging 
Table 4 Carbohydrate concentrations in the cytosol of mesophyll cells of leaves as well as in the apoplast and in the phloem sap from common plantain, sea plantain, peach and celery
Mean values of $n=3-9$ independent measurements are shown. Mean values \pm SD

\begin{tabular}{|c|c|c|c|c|c|}
\hline & \multicolumn{3}{|c|}{ Concentration (mM) } & \multirow{2}{*}{$\begin{array}{l}\text { Ratio } \\
\text { Phl/apo }\end{array}$} & \multirow{2}{*}{$\begin{array}{l}\text { Ratio } \\
\mathrm{Phl} / \mathrm{cyt}\end{array}$} \\
\hline & Cytosol & Apoplast & Phloem sap & & \\
\hline \multicolumn{6}{|l|}{ Common plantain } \\
\hline Sorbitol & $133 \pm 48$ & $5.5 \pm 1.2$ & $422 \pm 129$ & 77 & 3.2 \\
\hline Sucrose & $12 \pm 2.5$ & $0.3 \pm 0.1$ & $645 \pm 225$ & 2,150 & 40 \\
\hline Sorbitol/sucrose & 11 & 18 & 0.7 & & \\
\hline \multicolumn{6}{|l|}{ Sea plantain } \\
\hline Sorbitol & $13 \pm 8.4$ & $7.9 \pm 2.5$ & $315 \pm 105$ & 40 & 24 \\
\hline Sucrose & $20 \pm 4.9$ & $0.3 \pm 0.1$ & $355 \pm 112$ & 1,183 & 18 \\
\hline Sorbitol/sucrose & 0.7 & 26 & 0.9 & & \\
\hline \multicolumn{6}{|l|}{ Peach } \\
\hline Sorbitol & $290 \pm 62$ & $24.4 \pm 9.5$ & $582 \pm 90$ & 24 & 2.0 \\
\hline Sucrose & $106 \pm 16$ & $1.2 \pm 0.6$ & $207 \pm 51$ & 173 & 2.0 \\
\hline Sorbitol/sucrose & 2.7 & 20 & 2.8 & & \\
\hline \multicolumn{6}{|l|}{ Celery } \\
\hline Mannitol & $100 \pm 39$ & $6.7 \pm 3.3$ & $732 \pm 131$ & 109 & 7.3 \\
\hline Sucrose & $86 \pm 28$ & $1.2 \pm 0.9$ & $389 \pm 84$ & 324 & 4.5 \\
\hline Mannitol/sucrose & 1.2 & 5.6 & 1.9 & & \\
\hline
\end{tabular}

between 200 and $700 \mathrm{mM}$. In the two Plantaginaceae sucrose concentrations, measured directly, were slightly higher than sorbitol concentrations, whereas in peach and celery the sorbitol and mannitol concentrations, respectively, were 3- and 2-fold higher than the sucrose concentration. Therefore, the ratios of sugar alcohol to sucrose concentrations for the four plants differ between 0.7 in common plantain and 2.8 in peach. No or only traces of glucose and fructose were found in the phloem, and the concentration of myo-inositol was in the low millimolar range (data not shown).

Sorbitol and mannitol, respectively, dominated the apoplast of all four plants, while sucrose was present at concentrations at or below $1 \mathrm{mM}$ (Table 4). Nevertheless, the polyol as well as the sucrose concentrations in the apoplast were much lower than in phloem sap (Table 4). The concentration differences between the apoplast and the phloem were about 100-fold for mannitol, between 20- and 80-fold for sorbitol and between 170- and 2,200-fold for sucrose.

\section{Discussion}

Despite the central importance of the compartmentation of sugar alcohols in plant cells in relation to phloem transport, up to now the sugar alcohol distribution in subcellular compartments has been the subject of very few studies. We have used non-aqueous fractionation in addition to HPLC to study subcellular sugar alcohol distributions in common plantain, sea plantain, peach, and celery which translocate large amounts of sorbitol or mannitol within the phloem. It should be pointed out that the non-aqueous fractionation technique was developed for determination of subcellular concentrations of metabolites that are exclusively located in the mesophyll (Gerhardt and Heldt 1984). For other metabolites such as sugars, which are also present in tissues other than the mesophyll, the method tends to somewhat overestimate concentrations in the mesophyll cell compartments, because the amounts of metabolites in other tissues are assumed to be negligible. However, Voitsekhovskaja et al. (2006) have shown that concentrations of hexoses and sucrose in mesophyll cells measured either by single-cell technique or by non-aqueous fractionation were similar. A possible explanation is that e.g., the dense phloem tissue remains intact and will be removed from the other tissue during fractionation process (either in the sieve or in the bottom of the gradient; see chapter "Non-aqueous fractionation of leaf tissue").

Sorbitol and mannitol concentrations are high in stroma and cytosol despite of a large vacuolar polyol depot

The main proportion of sorbitol and mannitol was found in the vacuolar compartment (48-87\%; Fig. 1a-d) whereas in most species analyzed the highest concentrations were found in the stroma (Table 3 ). High concentrations of polyols in the stroma require either polyol-uptake or polyol-synthesis inside this compartment. Unfortunately, little is known about the intracellular distribution of the enzymes involved in sorbitol synthesis. Experiments with apple (Yamaki 1981) support a predominantly chloroplastic location for sorbitol-6-phosphate dehydrogenase. On the other hand, the intracellular immunocytochemical localization data for mannose-6-phosphate reductase, the key enzyme 
for mannitol synthesis, show that this enzyme is primarily cytosolic (Loescher and Everard 1996). So far no polyol transporter has been demonstrated to be localized in the envelopes of chloroplasts. The function of high concentrations of polyols in the stroma could be e.g., for osmoregulation because the concentration of several other metabolites or ions in this compartment is much lower than in the cytosol and in the vacuole (Table 3; Winter et al. 1993; Lohaus et al. 1998; Voitsekhovskaja et al. 2006).

Of the plants analyzed which produce sorbitol, only in sea plantain was the sorbitol concentration in the vacuole of mesophyll cells higher by about 7-fold than that in the cytosol (Table 3). The only previous report about subcellular distribution of sorbitol was of snapdragon (Moore et al. 1997) where up to $100 \%$ of sorbitol was found in the vacuole. However, the total leaf content of sorbitol was much lower in snapdragon than in plants in our study. A higher sorbitol concentration in the vacuole than in the cytosol, as in sea plantain, demonstrates that there is an active transport of sorbitol into the vacuole, because most likely sorbitol is either produced in the cytosol or in other cell compartments. So far no sorbitol transporter has been demonstrated to be localized in the tonoplast of mesophyll cells.

The high sorbitol concentration in the vacuoles of sea plantain indicates that sorbitol may have more functions in that species than just serving as a transport-form for carbon. Sea plantain is a more salt tolerant plant species than common plantain (Ahmad et al. 1979). In the study presented here no salt stress was given but the plants have taken up sodium from the soil used for plant culture. As a result the sodium content in common plantain leaves was $4.3 \pm 1.1 \mu \mathrm{mol}(\mathrm{g} \mathrm{FW})^{-1}$, whereas sea plantain contained $36 \pm 6.5 \mu \mathrm{mol}\left(\mathrm{g} \mathrm{FW}^{-1}\right.$ which corresponds to the higher sorbitol content in the leaves in sea plantain (Table 3). Stoop et al. (1996) have discussed that mannitol may accumulate in the vacuole of salt-tolerant plant species for redistribution to the cytosol in response to stress, and recently Pommerrenig et al. (2007) demonstrated increased sorbitol contents in leaf cells of common plantain in response to salt treatment.

In leaves of celery three quarters of mannitol was located in the vacuole (Fig. 1d). In A. barclaiana (Voitsekhovskaja et al. 2006), parsley and snapdragon (Moore et al. 1997) the vacuole, stroma and the cytosol contained each onethird of leaf mannitol, but the total leaf content of mannitol was much lower in these three plants than in celery. Probably the higher leaf content of mannitol requires the deposition of a greater portion of mannitol in the vacuole. Despite the high proportion of mannitol in the vacuole, cytosolic and stromal mannitol concentrations were calculated to be similar to be higher than those in the vacuole (Fig. 1d and Table 3). Mannitol is synthesized in the cytosol of leaf cells (Rumpho et al. 1983) and similar concentrations of mannitol in the vacuole and the cytosol indicate that diffusion of mannitol between both compartments could be responsible for the pattern observed. On the other hand, mannitol is transiently stored in the vacuoles of parenchyma cells of fleshy petioles (Greutert et al. 1998). Similarly, Keller and Matile (1989) estimated a 3-fold higher mannitol concentration in the vacuole than in the cytosol of petiole cells, suggesting that an active uptake system of mannitol is present in the tonoplast. The vacuolar mannitol concentration higher in storage petioles than in source leaves may be a reflection of the different functions of these two organs in celery.

\section{A large pool of myo-inositol is located in the stroma}

A great portion of myo-inositol is localized in the stroma (Fig. 1a-d) which corresponds to earlier findings (Voitsekhovskaja et al. 2006; Moore et al. 1997). The high proportion of myo-inositol in the stroma also resulted in the highest myo-inositol concentration in this compartment (8-15 mM, Table 3), compared to other compartments. This stromal pool probably originates from its synthesis by the stromal isoform of the myo-inositol synthesizing enzyme, myo-inositolphosphate synthase (Adhikari et al. 1987). In the halophyte, Mesembryanthemum crystallinum, myoinositol synthesis is increased in response to salt stress (Ishitani et al. 1996). Leaves of sea plantain contained a 8 -fold higher salt content than those of common plantain (see above). Despite this difference, the content of myoinositol was very similar in both plants in contrast to the sorbitol content (Table 3). Therefore, it seems for plantain that the sorbitol concentration is correlated with the sodium concentration rather than the myo-inositol concentration, even under low sodium conditions.

The subcellular distribution of hexoses and sucrose is similar in plant leaves with and without sugar alcohols

Hexoses are often confined to the vacuole (Riens et al. 1991; Winter et al. 1992). Figure 1a-d shows that these findings are also true for the four species analyzed here: concentrations of glucose and fructose were always higher in the vacuole than in the cytosol or in the stroma (Table 3). Both facilitated diffusion and active transport have been described for vacuolar uptake of glucose. Facilitated diffusion has been discussed for the transport of glucose across the tonoplast in celery (Daie 1987). Heineke et al. (1994) suggested that leaf vacuoles may contain transporters for the active uptake of hexoses. However, Martinoia et al. (2000) noted that accumulation of hexoses within the vacuole is not a proof for energized hexose uptake, because the vacuole contains acid invertases, allowing for enzymatic production of hexoses on the vacuoles. 
Between 50 and $80 \%$ of the sucrose detected in the four species analyzed were located in the vacuole (Fig. 1a-d). However, due to the smaller volume of the cytosol relative to that of the vacuolar compartment, cytosolic sucrose concentrations were calculated to be similar to or even higher than those in the vacuole (Table 3). A similar distribution has also been reported for leaves of other species (Riens et al. 1991; Winter et al. 1992). The lowest vacuolar sucrose concentration was found in the two Plantaginaceae and may be related to the overall low sucrose content in the leaves of these two species (Table 3). Recently Endler et al. (2006) have localized sucrose transporters in the tonoplast of barley (HvSUT2) and Arabidopsis (AtSUT4) and proposed that sucrose transporters are involved in vacuolar transport.

Concentration ratios between the cytosol of mesophyll cells and the phloem were similar for sucrose and sugar alcohols

Sugar alcohols and sucrose are the major compounds translocated in the four species analyzed. The phloem concentrations of sorbitol and mannitol (Table 4) were in the same range as that of sucrose (300-700 versus $200-700 \mathrm{mM}$ ) although the ratio of sorbitol, or mannitol, to sucrose varied between 0.7 and 2.8. High concentrations of sugar alcohols in the phloem indicate that a high proportion of assimilated carbon is exported in this way. Moing et al. (1997) estimated that sorbitol accounts for $60-90 \%$ of the carbon exported from the leaf in peach and in celery, while mannitol was shown to account for 10-60\% (Daie 1986).

In the four plants the sucrose concentration was always higher in the phloem sap than in the cytosol of mesophyll cells although the concentration ratio of sucrose between the cytosol of mesophyll cells and the phloem varied between 2 - and 40-fold (Table 4). The different ratios arose from variable cytosolic sucrose concentrations rather than by different phloem concentrations in the four plants (Table 4). With the exception of common plantain the ratios of both, sorbitol and mannitol, between the phloem and the cytosol of mesophyll cells were similar to the corresponding ratios of sucrose. In common plantain the overall sucrose concentration was relatively low which resulted in a higher ratio for sucrose than for sorbitol.

Concentration ratios between the apoplast and the phloem for sucrose and sugar alcohols with relation to the phloem loading mode

Gamalei (1989) has shown that the members of Plantaginaceae display a virtually complete symplastic isolation of the sieve element-companion cell complex, as indicated by a very low plasmodesmal abundance between phloem companion cells and bundle-sheath cells. Sucrose and sorbitol were present in the apoplast of both Plantaginaceae, however, the concentrations were much lower than in phloem sap (Table 4). Therefore, the loading of both sucrose and sorbitol into the phloem must be energized. This is consistent with the observation that sucrose transporters (PmSUC1, PmSUC2, and PmSUC3; Gahrtz et al. 1994; Barth et al. 2003) as well as polyol transporters (PmPLT1 and PmPLT2; Ramsperger-Gleixner et al. 2004) were cloned from $P$. major. The function of the putative transporter proteins in phloem loading was corroborated by their specific localization in companion cells of source leaf phloem (PmSUC2, Stadler et al. 1995; PmPLT1 and PmPLT2 Ramsperger-Gleixner et al. 2004). The $K_{\mathrm{m}}$ value of PmSUC2 for sucrose was about $1 \mathrm{mM}$ (Gahrtz et al. 1994) and the $K_{\mathrm{m}}$ value of PmPLT1 for sorbitol was about $12 \mathrm{mM}$ (Ramsperger-Gleixner et al. 2004), which are in the same range as the apoplastic sucrose and sorbitol concentrations (Table 4). No data of transporters exists for sea plantain, but the concentration gradients for sucrose as well as those for sorbitol strongly suggest active transport systems for both carbohydrates. Together, the data support apoplastic transfer of sucrose and sorbitol in the members of Plantaginaceae.

Pathway and mechanism of phloem loading in peach are still a matter of debate (Moing et al. 1997). The phloem structures of Rosaceae trees were shown to be of the intermediate-type (moderate numbers of plasmodesmal connections between mesophyll and companion cells; Gamalei 1989). Therefore, the mechanism of phloem loading in Prunus species could be symplastic, apoplastic or mixed. In apoplastic loaders, sucrose or sorbitol destined for phloem transport has to be released from the mesophyll cells into the apoplast, whereas in symplastic loaders, sugars and sugar alcohols synthesized in the mesophyll and destined for the phloem transport, are expected to stay in the symplast. Thus, it could be expected that the percentage of these carbohydrates in the cytosol of mesophyll cells in putative symplastic loaders would be much higher than in apoplastic loaders. Sorbitol concentrations were high in the phloem sap of peach (Table 4, Moing et al. 1997) and in addition to sucrose sorbitol is in general a major photoassimilate translocated in the phloem of woody Rosaceae (Bieleski 1982). The concentration ratios for sucrose and sorbitol between the cytosol of mesophyll cells and the phloem were about 2-fold for each carbohydrate (Table 4). These are the lowest ratios among the species analyzed and much lower than those determined for apoplastic phloem loaders (ratio of sucrose 5-30; Lohaus et al. 1995; Lohaus and Möllers 2000). A 2-fold concentration ratio for sucrose corresponds to the gradient found in Alonsoa meridionalis, a Scrophulariaceae with a symplastic phloem loading mode (Knop et al. 2001). A lower concentration ratio is obviously easier to balance or even reverse, depending on the 
metabolic situation, than a steeper ratio. Therefore, the possibility of phloem loading of sucrose and sorbitol by simple diffusion cannot be ruled out. Turgeon and Medville (1998) were unable to detect accumulation of sucrose against the concentration gradient in minor veins of willow leaves, representing another woody species.

On the other hand, leaf infiltration with $p$-chloromercuribenzenesulfonic acid (PCMBS) was found to inhibit sugar phloem transport in peach and Moing et al. (1992) further concluded from this result that apoplastic phloem loading predominates in peach. Unfortunately up to now no sucrose or sorbitol transporters were cloned from peach as they had from other Rosaceae (Prunus cerasus, Gao et al. 2003; Malus domestica, Watari et al. 2004). Watari et al. (2004) reported the existence of three sorbitol transporters in apple source leaves with apparent $K_{\mathrm{m}}$ values for sorbitol between 0.7 and $3.2 \mathrm{mM}$. The apoplastic sorbitol concentration in apple leaves was not detected but the corresponding concentration in peach was about 25 -fold higher (Table 4).

Sucrose and sorbitol uptake in plasma membrane vesicles from peach leaves exhibited saturated kinetics (Marquat et al. 1997). These results suggest that uptake of sorbitol and sucrose is carrier mediated. Active absorption of sucrose was completely inhibited by PCMBS, contrary to the absorption of sorbitol (Marquat et al. 1997). The activities of sorbitol transporters from Rosaceae heterologously expressed in yeast were also not or only slightly inhibited by PCMBS (Gao et al. 2003; Watari et al. 2004). Ramsperger-Gleixner et al. (2004) have shown that sorbitol transport by PmPLT1, but not that driven by PmPLT2, is inhibited by PCMBS. Therefore, PCMBS sensitivity of sorbitol transport can vary and the insensitivity of transport processes against PCMBS conclusively does not rule out that carriers are involved.

Unfortunately, the type of phloem loading in peach is still an open question; the available data are consistent with either an apoplastic or a symplastic mode of loading or, more likely, a combination of both.

Celery is the only plant species analyzed here which translocates mannitol in addition to sucrose. The concentration ratio of sucrose between the apoplast and the phloem sap was about 300 -fold and the ratio for mannitol was about 100-fold (Table 4). Studies of metabolite uptake into plasma membrane vesicles from phloem tissues of celery petioles by Salmon et al. (1995) showed mannitol- as well as sucrose-proton-co-transport, indicating that in this species mannitol and sucrose transporters may be involved in phloem loading. Noiraud et al. (2000) have cloned two sucrose transporters from celery (AgSUT1, AgSUT2). AgSUT1 is expressed in mature leaves and phloem of petioles. Noiraud et al. (2001) have also cloned a cDNA of a mannitol transporter (AgMaT1) of celery which is not sensitive to PCMBS. The expression profile for AgMaT1 in source leaves and phloem was in agreement with a role in phloem loading of mannitol in celery. The AgMaT1 protein expressed in yeast cells exhibited a $K_{\mathrm{m}}$ value for mannitol uptake of about $0.3 \mathrm{mM}$ (Noiraud et al. 2001). The apoplastic mannitol concentration was 25 -fold higher (Table 4). In summary, celery shows all characteristics of a typical apoplastic phloem loader.

The comparison of common plantain, sea plantain, peach, and celery indicates that different modes of phloem loading are employed by different plant families, which may in part be related to the respective ecophysiological requirements.

Acknowledgments The authors like to thank Melanie Klenke for excellent technical assistance and Ingo Heilmann for critical reading of the manuscript. This work was supported by the Deutsche Forschungsgemeinschaft (DFG).

Open Access This article is distributed under the terms of the Creative Commons Attribution Noncommercial License which permits any noncommercial use, distribution, and reproduction in any medium, provided the original author(s) and source are credited.

\section{References}

Adhikari J, Bhaduri TJ, DasGupta S, Majunder AL (1987) Chloroplast as a locale of L-myo-inositol-1-phosphate synthase. Plant Physiol 85:611-614

Ahmad I, Larher F, Stewart GR (1979) Sorbitol, a compatible osmotic solute in Plantago maritima. New Phytol 82:671-678

Barth I, Meyer S, Sauer N (2003) PmSUC3: characterization of a SUT2/SUC3-type sucrose transporter from Plantago major. Plant Cell 15:1375-1385

Bieleski RL (1982) Sugar alcohols. In: Loewus, Tanner W (eds) Encyclopedia of plant physiology, N.S., vol 13A: Plant carbohydrates. Springer, Berlin, pp 158-192

Daie J (1986) Kinetics of sugar transport in isolated vascular bundles and phloem tissue of celery. J Am Soc Hortic Sci 111:216-220

Daie J (1987) Sucrose uptake in isolated phloem of celery is a single saturable transport system. Planta 171:474-482

Delesse MA (1847) Procédé mecanique pour determiner la composition des roches. C R Acad Sci (Paris) 25:544

Endler A, Meyer S, Schelbert S, Schneider T, Weschke W, Peters SW, Keller F, Baginsky S, Martinoia E, Schmidt UG (2006) Identification of a vacuolar sucrose transporter in barley and Arabidopsis mesophyll cells by a tonoplast proteomic approach. Plant Physiol 141:196-207

Farré EM, Tiessen A, Roessner U, Geigenberger P, Tretheway RN, Willmitzer L (2001) Analysis of the compartmentation of glycolytic intermediates, nucleotides, sugars, organic acids, amino acids, and sugar alcohols in potato tubers using a nonaqueous fractionation method. Plant Physiol 127:685-700

Flora LL, Madore MA (1993) Stachyose and mannitol transport in olive (Olea europaea. L.). Planta 189:484-490

Gahrtz M, Stolz J, Sauer N (1994) A phloem-specific sucrose- $\mathrm{H}^{+}$symporter from Plantago major L. supports the model of apoplastic phloem loading. Plant J 6:697-706

Gamalei Y (1989) Structure and function of leaf minor veins in trees and herbs. Trees 3:96-110

Gao Z, Mauroussed L, Lemoine R, Yoo S-D, van Nocker S, Loescher W (2003) Cloning, expression, and characterization of sorbitol 
transporters from developing sour cherry fruit and leaf sink tissue. Plant Physiol 131:1566-1575

Gerhardt R, Heldt HW (1984) Measurements of subcellular metabolite levels by fractionation of freeze-stopped material in nonaqueous media. Plant Physiol 75:542-547

Greutert H, Martinoia E, Keller F (1998) Mannitol transport by vacuoles of storage parenchyma of celery petioles operates by facilitated diffusion. J Plant Physiol 153:91-96

Heineke D, Wildenberger K, Sonnewald U, Willmitzer L, Heldt H-W (1994) Accumulation of hexose in leaf vacuoles: studies with transgenic tobacco plants expressing yeast-derived invertase in the cytosol, vacuole or apoplasm. Planta 194:29-33

Ishitani M, Majumder AL, Bornhouser A, Michalowski CB, Jensen RG, Bohnert HJ (1996) Coordinate transcriptional induction of myoinositol metabolism during environmental stress. Plant J 9:537-548

Keller F, Matile P (1989) Storage of sugars and mannitol in petioles of celery leaves. New Phytol 113:291-299

Knop C, Voitsekhovskaja O, Lohaus G (2001) Sucrose transporters in two Scrophulariaceae with different types of transport sugar. Planta 213:80-89

Leidreiter K, Kruse A, Heineke D, Robinson DG, Heldt H-W (1995) Subcellular volumes and metabolite concentrations in potato (Solanum tuberosum cv. Désirée) leaves. Bot Acta 108:439-444

Loescher WH, Everard JD (1996) Sugar alcohol metabolism in sink and source. In: Zamski E, Schaffer AA (eds) Photoassimilate distribution in plants and crops. New York, pp 185-207

Loewus FA, Loewus MW (1983) myo-Inositol: its biosynthesis and metabolism. Annu Rev Plant Physiol 34:137-161

Lohaus G, Möllers C (2000) Phloem transport of amino acids in two Brassica napus genotypes and one Brassica carinata in relation to their seed protein content. Planta 211:833-840

Lohaus G, Winter H, Riens B, Heldt HW (1995) Further studies of the phloem loading process in leaves of barley and spinach: comparison of metabolite concentrations in the apoplastic compartment with those in the cytosolic compartment and in the sieve tubes. Bot Acta 3:270-275

Lohaus G, Büker M, Hußmann M, Heldt H-W (1998) Transport of amino acids with special emphasis on the synthesis and transport of asparagine in Illinois low protein and Illinois high protein strains of maize. Planta 205:181-188

Lohaus G, Pennewiss K, Sattelmacher B, Hussmann M, Muehling KH (2001) Is the infiltration-centrifugation technique appropriate for the isolation of apoplastic fluid? A critical evaluation with different plant species. Physiol Plant 111:457-65

Marquat C, Pétel G, Gendraud M (1997) Saccharose and sorbitol transporters from plasmalemma membrane vesicles of peach tree leaves. Biol Plant 39:369-378

Martinoia E, Massonneau A, Frangne N (2000) Transport processes of solutes across the vacuolar membrane of higher plants. Plant Cell Physiol 41:1175-1186

Moing A, Carbonne F, Rashad MH, Gaudillère JP (1992) Carbon fluxes in mature peach leaves. Plant Physiol 100:1878-1884

Moing A, Carbonne F, Zipperlin B, Svanella L, Gaudillère JP (1997) Phloem loading in peach: symplastic or apoplastic? Physiol Plant 101:489-496

Moore BD, Palmquist DE, Seemann JR (1997) Influence of plant growth at high $\mathrm{CO}_{2}$ concentrations on leaf content of ribulose-1,5bisphosphate carboxylase/oxygenase and intracellular distribution of soluble carbohydrates in tobacco, snapdragon, and parsley. Plant Physiol 115:241-248
Negm FB, Loescher WH (1981) Characterization and partial purification of aldose-6-phosphate reductase (alditol-6-phosphate:NADP 1-oxidoreductase) from apple leaves. Plant Physiol 67:139-142

Noiraud N, Delrot S, Lemoine R (2000) The sucrose transporter of celery. Identification and expression during salt stress. Plant Physiol 122:1447-1455

Noiraud N, Maurousset L, Lemoine R (2001) Identification of a mannitol transporter, AgMaT1, in celery phloem. Plant Cell 13:695705

Pommerrenig B, Stal Papini-Terzi F, Sauer N (2007) Differential regulation of sorbitol and sucrose loading into the phloem of $P$. $m a-$ jor in response to salt stress. Plant Physiol 144:1029-1038

Ramsperger-Gleixner M, Geiger D, Hedrich R, Sauer N (2004) Differential expression of sucrose transporter and polyol transporter genes during maturation of common plantain companion cells. Plant Physiol 134:147-160

Riens B, Lohaus G, Heineke D, Heldt H-W (1991) Amino acid and sucrose content determined in the cytosolic, chloroplastic and vacuolar compartment and in the phloem sap of spinach leaves. Plant Physiol 97:227-233

Rumpho ME, Edwards GE, Loescher WH (1983) A pathway for photosynthetic carbon flow to mannitol in celery leaves. Activity and localization of key enzymes. Plant Physiol 73:869-873

Salmon S, Lemoine R, Jamai A, Bouché-Pillon S, Fromont JC (1995) Study of sucrose and mannitol transport in plasma-membrane vesicles from phloem and non-phloem tissues of celery (Apium graveolens $\mathrm{L}$.) petioles. Planta 197:76-83

Stadler R, Brandner L, Schulz A, Gahrtz M, Sauer N (1995) Phloem loading by the PmSUC2 sucrose carrier from Plantago major occurs into companion cells. Plant Cell 7:1545-1554

Stoop JMH, Williamson JD, Pharr DM (1996) Mannitol metabolism in plants: a method for coping with stress. Trends Plant Sci 1:139 144

Turgeon R, Medville R (1998) The absence of phloem loading in willow leaves. Proc Natl Acad Sci USA 95:12055-12060

Voitsekhovskaja OV, Koroleva OA, Batashev DR, Tomos AD, Gamalei YV, Heldt H-W, Lohaus G (2006) Phloem loading models of two Scrophulariaceae species with either extensive or moderate symplastic continuity between mesophyll and phloem. Plant Physiol 140:383-395

Watari J, Kobae Y, Yamaki S, Yamada K, Toyofuku K, Tabuchi T, Shiratake K (2004) Identification of sorbitol transporters expressed in the phloem of apple source leaves. Plant Cell Physiol 45:1032-1041

Winter H, Lohaus G, Heldt H-W (1992) Phloem transport of amino acids in relation to their cytosolic levels in barley leaves. Plant Physiol 99:996-1004

Winter H, Robinson DG, Heldt H-W (1993) Subcellular volumes and metabolite concentrations in barley leaves. Planta 191:180-190

Winter H, Robinson DG, Heldt H-W (1994) Subcellular volumes and metabolite concentrations in spinach leaves. Planta 193:530-535

Yamaki S (1981) Subcellular localization of sorbitol-6-phosphate dehydrogenase in protoplast from apple cotyledons. Plant Cell Physiol 22:359-367

Zimmermann MH, Ziegler H (1975) List of sugars and sugar alcohols in sieve-tube exudates. In: Zimmermann MH, Milburn JA (eds) Encyclopedia of plant physiology, vol. 1: transport in plants, Springer, Berlin, pp 479-503 